\title{
Necessidades do cuidador familiar no cuidado à pessoa dependente: uma revisão integrativa da literatura
}

Family caregiver's needs in caring for the dependent person: an integrative literature review Necesidades del cuidador familiar en relación al cuidado de la persona dependiente: una revisión integradora de la literatura

Ricardo Manuel da Costa Melo*; Marília dos Santos Rua**; Célia Samarina Vilaça de Brito Santos***

\section{Resumo}

Contexto: O envelhecimento e o aumento da dependência da população implicam a necessidade crescente de cuidados no domicílio a serem prestados por familiares.

Objetivos: Determinar quais as principais necessidades manifestadas pelo cuidador familiar no cuidado à pessoa dependente, através de uma revisão da literatura.

Metodologia: Recorreu-se à pesquisa em bases de dados eletrónicas, nacionais e internacionais, com o recurso a motores de busca específicos, tendo sido obtido inicialmente um total de 516 produções científicas que, após a análise segundo os critérios de inclusão, ficou resumido a um conjunto de 21 artigos.

Resultados: Da análise da evidência científica obtida, esta orienta para um conjunto de necessidades, as quais agrupamos à luz da Teoria das Transições: recursos comunitários e sociais, conhecimentos e preparação, significado pessoal, crenças e atitudes e condição socioeconómica.

Conclusão: Podemos concluir que o correto diagnóstico das necessidades dos cuidadores familiares é determinante para o planeamento eficaz das intervenções de enfermagem, o que pode traduzir-se em ganhos para a saúde, tanto do cuidador, como da pessoa que é cuidada.

Palavras-chave: cuidadores familiares; determinação de necessidades de cuidados de saúde; membros da família; idoso dependente.

\begin{abstract}
Background: Population ageing and dependency increase require a growing need of home-based care to be provided by family members.

Objectives: To determine what are the main needs expressed by family caregivers in caring for the dependent person by performing a literature review.

Methodology: Search in both national and international electronic databases, using specific search engines. A total of 516 scientific studies were initially obtained. After an analysis according to inclusion criteria, a total of 21 papers were selected.

Results: The obtained scientific evidence highlights certain needs, which were divided according to the Transitions Theory: community and societal resources, knowledge and preparation, personal meaning, beliefs and attitudes, and socioeconomic status.

Conclusion: We can conclude that the correct diagnosis of family caregivers' needs is essential for an effective planning of nursing interventions, which can be translated into health gains both for the caregiver and the patient.
\end{abstract}

Keywords: family caregivers; needs assessment; family; frail elderly.

\footnotetext{
* Licenciatura em Enfermagem e Doutorando em Ciências de Enfermagem (Instituto Ciências Biomédicas Abel Salazar, Universidade do Porto, Enfermeiro, Centro Médico da Praça Lda, 4520-409 Santa Maria da Feira, Portugal [rmcmelo@hotmail.com]. Morada de correspondência: Zona Industrial de Masteirô, Casa 2, 4520-409, Santa Maria da Feira, Portugal.

** Doutorada em Ciências da Saúde; Universidade de Aveiro. Professora Adjunta, Escola Superior de Saúde da Universidade de Aveiro, 3810-159 Sta Joana - Aveiro, Portugal [mrua@ua.pt].

*** Doutorada em Psicologia. Professora Coordenadora, Escola Superior de Enfermagem do Porto, 4425 - 011, Águas Santas-Maia, Portugal [celiasantos@esenf.pt].
}

\section{Resumen}

Contexto: El envejecimiento y el aumento de la dependencia de la población implican una creciente necesidad de que los familiares presten cuidados en el hogar.

Objetivos: Determinar cuáles son las principales necesidades manifestadas por los cuidadores familiares en relación al cuidado de la persona dependiente, a través de una revisión de la literatura. Metodología: Se realizó una búsqueda en bases de datos electrónicas, nacionales e internacionales, y se usaron motores de búsqueda específicos. Inicialmente se obtuvo un total de 516 trabajos científicos que, después del análisis de acuerdo con los criterios de inclusión, se resumió en 21 artículos científicos.

Resultados: El análisis de la evidencia científica obtenida conduce hacia un conjunto de necesidades, que agrupamos de acuerdo con la Teoría de la Transición: recursos comunitarios y sociales, conocimiento y habilidades, significado personal, creencias y actitudes, y nivel socioeconómico.

Conclusión: Un diagnóstico correcto de las necesidades de los cuidadores es crucial para la planificación efectiva de las intervenciones de enfermería, lo cual puede resultar en beneficios para la salud, tanto para el cuidador como para la persona cuidada.

Palabras clave: los cuidadores familiares; evaluación de necesidades; familia; anciano frágil.

Recebido para publicação em: 10.01 .14

Aceite para publicação em: 28.03 .14 


\section{Introdução}

O envelhecimento populacional é uma evidência atual, com sérias consequências para a sociedade. Segundo o Instituto Nacional de Estatística (2012), no recenseamento de 2011, denota-se um agravamento deste envelhecimento que se fica a dever à melhoria dos cuidados de saúde, refletido no aumento da esperança média de vida das pessoas, mas também devido ao aumento da incidência das doenças crónicas e/ou incapacitantes. Isto evidencia o aumento do topo da pirâmide etária e, associado a este, uma diminuição da base da mesma, resultado da diminuição da taxa da natalidade (Carrilho \& Patrício, 2008). A diminuição das capacidades, natural com o avançar do ciclo vital e o aumento das doenças crónicas conduzem a uma perda de autonomia e aumento da dependência de terceiros para a satisfação das mais elementares necessidades diárias e manutenção da qualidade de vida (Sequeira, 2010).

A prestação destes cuidados pode ser extremamente desgastante e, normalmente, acarreta um aumento do stress e da sobrecarga por parte de quem os exerce. Por outro lado, os cuidadores enfrentam um conjunto de desafios constantes, pelo que necessitam de se organizar e preparar adequadamente, para assim a vivenciarem de forma saudável a transição para este novo papel. Neste sentido, torna-se importante a coordenação com os profissionais de saúde, designadamente os enfermeiros, para satisfazerem as suas necessidades, manterem o adequado nível de saúde e bem-estar e, de igual modo, garantirem a continuidade nos cuidados prestados à pessoa dependente.

Agente facilitador desta continuidade é a rede de cuidados continuados, que pode ser um importante apoio para estes cuidadores, quando se articula devidamente com os diferentes serviços de saúde. No nosso país, os cuidados continuados são constituídos por uma rede de apoio formal e uma de apoio informal (Martins, 2008). O papel de membro da família prestador de cuidados ou cuidador familiar é fundamental e de extrema importância na rede de apoio informal, pois tem como objetivo assegurar a continuidade dos cuidados ao seu familiar dependente. Neste âmbito, considera-se o cuidado informal todo aquele que não é remunerado, prestado de modo parcial ou integral, à pessoa com dependência, por pessoas pertencentes, em geral, à rede familiar (Sarmento, Pinto, \& Monteiro, 2010). O cuidado informal pode ser assumido por amigos, vizinhos ou outros mas, normalmente, é a família que assume a responsabilidade da organização ou assistência e prestação de cuidados.

A família desempenha, desde os tempos mais remotos, um importante papel para a pessoa, pelo que é considerada um sistema social primário, dentro do qual o indivíduo é cuidado e se desenvolve a nível físico, pessoal e emocional (Sarmento et al., 2010). Neste contexto, desempenha, desde há muito, uma função essencial no acompanhamento e apoio aos membros incapacitados e dependentes de terceiros, por doença ou velhice, constituindose como o grupo primário de apoio e tendo um papel primordial no cuidado a longo prazo (Sequeira, 2010). Quando a família é confrontada com a necessidade de cuidar de um dos seus elementos que manifeste dependência nas suas atividades diárias, o que acontece frequentemente, as tarefas que advêm da atividade de cuidar não são distribuídas equitativamente por todos os seus elementos. A prestação de cuidados é um processo árduo, complexo e dinâmico, caracterizado por constantes variações ao longo do tempo, tanto nas necessidades como nos sentimentos de quem presta os cuidados e os recebe (Sarmento et al., 2010). Cuidar de uma pessoa dependente pode traduzir-se num trabalho desgastante e exigente, tanto do ponto de vista físico como afetivo, pelo que requer uma grande disponibilidade de tempo e energia. O papel de cuidador informal é, maioritariamente assumido por familiares, pelo que é compreensível designálo por cuidador familiar ou membro da família prestador de cuidados, responsável pela prevenção ou tratamento da doença ou incapacidade e organização de prestação de cuidados (International Council of Nursing, 2011). Os cuidadores familiares são, geralmente, mulheres, solteiras, domésticas ou desempregadas e que coabitam com a pessoa dependente.

Ao iniciar o desempenho destas novas funções, ocorre uma transição para o novo papel de cuidador familiar que, para além de ser um processo complexo, envolve inúmeras variáveis com influência mútua entre si e, consequentemente, com valores preditivos diferentes, em função de todas as que estão envolvidas, designadamente do cuidador, da pessoa dependente, 
do contexto e do ambiente em que se desenvolve a prestação de cuidados (Sequeira, 2010). Tratando-se de uma transição entendemos que, para o melhor entendimento deste processo e do modo como afeta os cuidadores familiares, é uma mais-valia o recurso a uma teoria de médio alcance, mais especificamente, a Teoria das Transições de Meleis.

Segundo esta teoria, a transição é caraterizada por diferentes etapas dinâmicas, marcos e pontos de viragem e pode ser definida através dos indicadores de processo e dos resultados finais (Meleis, 2010), sendo entendida como a passagem entre duas fases da vida, ou de um estado para outro, pelo que se espera alcançar a adequada adaptação ao novo papel e/ou situação. É, portanto, um conceito múltiplo que contém os elementos do processo, intervalo de tempo e perceção (Chick \& Meleis, 2010). Neste pressuposto existem fatores que podem funcionar como facilitadores ou inibidores de uma transição eficaz, tais como os conhecimentos e capacidades, o significado pessoal, as crenças e atitudes, a condição socioeconómica e os recursos comunitários e sociais disponíveis. Assim, as respostas dos cuidadores a todo este processo, o seu envolvimento, a confiança evidenciada e as estratégias de coping a que estes recorrem são indicadores de processo, que se esperam adequados, para que os indicadores de resultado (mestria e integração do novo papel) sejam os mais eficientes e saudáveis possíveis. Segundo a Teoria das Transições, os cuidadores familiares experienciam uma transição do tipo situacional (Schumacher \& Meleis, 2010). Este tipo de transição inclui situações inesperadas que ocorrem na vida da pessoa e requerem uma redefinição dos seus papéis e/ou integração de novos.

A transição está presente em diferentes domínios, entre os quais os cuidados de saúde. Neste âmbito, os enfermeiros são profissionais de saúde que, devido à sua atividade e à proximidade com as pessoas, lidam frequentemente com diversos processos de transição em contextos distintos e são, em inúmeras ocasiões, os principais cuidadores das pessoas em transição. Deste modo, assistem às mudanças e exigências que advêm das necessidades dos familiares na atividade de prestar cuidados e na assunção do papel de cuidador sendo, neste domínio, os profissionais de saúde que os prepararam para esta nova etapa, promovendo e incentivando a aquisição de novos conhecimentos e aprendizagem de habilidades e capacidades relacionadas com a situação vivenciada, para facilitarem todo o processo e facultarem uma melhor adaptação (Meleis, 2010).

Durante esta transição e considerando um conjunto de variáveis inerentes à prestação de cuidados, designadamente os seus recursos pessoais e sociais, capacidades, conhecimentos e a sua disponibilidade, os cuidadores familiares evidenciam um conjunto de necessidades, as quais devem ser focos privilegiados da atenção e ação interventiva dos enfermeiros. Estes profissionais devem então orientar as suas intervenções no sentido de satisfazer devidamente estas mesmas necessidades e, desta forma, obterem os ganhos para a saúde desejados e o bem-estar dos cuidadores.

Em suma, não só é importante o estabelecimento de parcerias entre cuidadores formais e informais, como também o planeamento de intervenções contextualizadas que visem facilitar e melhorar a qualidade dos cuidados prestados.

A prestação de cuidados e as consequências que advêm desta atividade tem sido alvo de estudo por parte de vários investigadores, pelas repercussões individuais, familiares e sociais que resultam deste. Assim, num estudo realizado por Sequeira (2010), este realça que os cuidadores se deparam com dificuldades a nível dos problemas relacionais, das restrições sociais, das reações ao cuidar e do apoio familiar. Cruz, Loureiro, Silva, e Fernandes (2010), numa revisão sobre as vivências do cuidador informal do idoso dependente, realça as dificuldades manifestadas por estes, designadamente: exigência do cuidado, insuficiência das respostas formais e informais, problemas financeiros e restrições sociais. Estas dificuldades, quando não são satisfeitas, podem traduzir-se em consequências nefastas para os cuidadores, designadamente, ansiedade, stress e/ ou sobrecarga, com consequências para os próprios, família e sociedade.

Com esta revisão da literatura pretendemos identificar e categorizar as necessidades mais evidenciadas pelos cuidadores familiares, emergentes do conhecimento científico produzido.

Com o objetivo de sintetizar o conhecimento atual sobre a temática, elaborou-se a seguinte questão de investigação: Quais as principais necessidades do cuidador familiar na transição para a prestação de cuidados à pessoa com dependência, evidenciadas pela literatura? 


\section{Procedimentos Metodológicos de Revisão Integrativa}

Tendo por base a questão de investigação supra mencionada e, tendo em consideração os conhecimentos que se pretendia sintetizar, realizouse uma revisão da literatura, segundo os descritores: Caregiver, Family Caregivers, Needs, Dependent. Utilizaram-se os carateres boleanos and e or para conjugar os diferentes descritores.

Os trabalhos encontrados foram classificados mediante o nível de evidência demonstrada, tendo sido classificados segundo a classificação proposta por Lewin, Singleton, e Jacobs (2008).

A pesquisa bibliográfica foi realizada entre abril e junho de 2011. Foram selecionados trabalhos publicados entre janeiro 2000 e junho de 2011, utilizando o inglês como idioma de eleição. Para o efeito, recorreu-se à pesquisa em bases de dados eletrónicas, nacionais e internacionais, com o recurso a motores de busca específicos, nas bases de dados disponíveis na área da saúde, designadamente a EBSCO [Cinahl, Medline, Mediclatina, ERIC, Psychology and Behavioral Sciences Collection, Cochrane Central Register of Controlled Trials, Database of Abstracts of Reviews of Effects, Cochrane Database of Systematic Reviews, Health Tecnology Assessments e Cochorane Methodology Register] e B-on [Annual Reviews, Current Contents (ISI), Elsevier - Science Direct (Freedom Collection), Nature, PubMed, RCAAP, SpringerLink (Springer) Kluwer), Taylor \& Francis, Web of Science (ISI) $e$
Wiley Online Library (Wiley)], sendo que a base de dados Academic Search Complete foi pesquisada por duas ocasiões, através de ambos os motores de busca. Foram incluídos trabalhos científicos que abordavam cuidadores familiares com idade superior a 18 anos, que cuidavam de pessoas com dependência no autocuidado no domicílio, com alusão às suas necessidades, no âmbito dos cuidados continuados. Utilizamos como critério de exclusão todos os trabalhos cuja temática fosse centrada nos cuidadores formais, nos cuidadores familiares com menos de 18 anos e cuidados prestados a nível institucional e/ou outras temáticas.

Inicialmente foi realizado o levantamento das necessidades mais evidenciadas na literatura científica através de, primeiramente, da leitura do título, seguido da leitura do resumo e, finalmente, numa fase mais adiantada da seleção dos trabalhos, da leitura integral do artigo selecionados. As necessidades realçadas pelos diversos autores foram agrupadas em categorias, elaboradas segundo a Teoria das Transições.

\section{Resultados e Interpretação}

Das quinhentas e dezasseis produções científicas obtidas inicialmente e considerando os critérios de inclusão e exclusão previamente definidos, obtivemos um conjunto de vinte e um artigos que se apresentam na Tabela 1 e que foram analisados criteriosamente, de modo a percecionar as principais necessidades demonstradas pelo cuidador.

\section{TABELA 1}

Apresentação dos artigos encontrados que cumprem critérios de inclusão

\begin{tabular}{|c|c|c|}
\hline Referência Bibliográfica & Tipo de Estudo & Nível de Evidência \\
\hline $\begin{array}{l}\text { Casado, B. L. (2008). Sense of need for financial support and respite services among } \\
\text { informal caregivers of older Americans. Journal of Human Behavior in the } \\
\text { Social Environment, } 18(3), 269-287 \text {. }\end{array}$ & Quantitativo & IV \\
\hline $\begin{array}{l}\text { Carretero, S., Garcés, J., \& Ródenas, F. (2006). Evaluation of the home help service and } \\
\text { its impact on the informal caregiver's burden of dependent elders. Internatio- } \\
\text { nal Journal of Geriatric Psychiatry, 22(8), 738-749. }\end{array}$ & Quantitativo & II \\
\hline $\begin{array}{l}\text { Robison, J., Fortinsky, R., Kleppinger, A., Shugrue, N., \& Porter, M. (2009). A broader } \\
\text { view of family caregiving: Effects of caregiving and caregiver conditions on de- } \\
\text { pressive symptoms, health, work, and social isolation. The Journals of Geron- } \\
\text { tology. Series B, Psychological Sciences and Social Sciences, 64(6), 788-798. }\end{array}$ & Quantitativo & II \\
\hline $\begin{array}{l}\text { Salin, S., Kaunonen, M., \& Astedt-Kurki, P. (2009). Informal carers of older family mem- } \\
\text { bers: How they manage and what support they receive from respite care. Jour- } \\
\text { nal of Clinical Nursing, 18(4), 492-501. }\end{array}$ & Quantitativo & IV \\
\hline $\begin{array}{l}\text { Woolfe, P., McMillan, M., \& Conway, J. (2007). The needs of caregivers of people with } \\
\text { COPD: A study. Australian Journal of Primary Health, 13(1), 28-35. }\end{array}$ & Quantitativo & IV \\
\hline
\end{tabular}


Francis, L. E., Bowman, K. F., Kypriotakis, G., \& Rose, J. H. (2011). Relationships and emotional wellbeing among African American and White advanced cancer caregivers. Patient Education and Couseling, 85(3), 446-453.

Ryn, M. van, Sanders, S., Kahn, K., Houtven, C. van, Griffin, J. M., Martin, M.,...Rowland, J. (2011). Objective burden, resources, and other stressors among informal cancer caregivers: A hidden quality issue? Psycho-Oncology, 20(1), 44-52.

Nichols, L. O., Martindale-Adamsb, J., Greeneb, W. A., Burnsc, R., Graneyb, M. J., \& Lummusb, A. (2009). Dementia caregivers' most pressing concerns. Clinical Gerontologist, 32(1), 1-14.

Sinclair, A. J., Armes, D. G., Randhawa, G., \& Bayer, A. J. (2010). Caring for older adults with diabetes mellitus: Characteristics of carers and their prime roles and responsibilities. Diabetic Medicine, 27(9), 1055-1059.

Ganea, L. W., Iosifb, A. M., Flynn-Wilsonc, L., Venturinod, M., Hagermanae, R. J., \& Seritanaf, A. L. (2010). Assessment of patient and caregiver needs in fragile Xassociated tremor/ataxia syndrome by utilizing Q-sort methodology. Aging $\mathcal{E}$ Mental Health, 14(8), 1000-1007.

Jullamate, P., de Azeredo, Z., Pául, C., \& Subgranon, R. (2006). Thai stroke patient caregivers: Who they are and what they need. Cerebrovasc Diseases, 21(1-2), 128-133.

Mavundla, T. R., Toth, F., \& Mphelane, M. L. (2009). Caregiver experience in mental illness: A perspetive from a rural community in South Africa. International Journal of Mental Health Nursing, 18(5), 357-367.

Davey, C., Wiles, R., Asburn, A., \& Murphy, C. (2004). Falling in Parkinson's disease: The impact on informal caregivers. Disability and Rehabilitation, 26(23), 13601366.

Molassiotis, A., Wilson, B., Blair, S., Howe, T., \& Cavet, J. (2009). Living with multiple myeloma: Experiences of patients and their informal caregivers. Support Care Cancer, 19(1), 101-111.

Chan, W. C., Ng, C., Mok, C. C., Wong, F. L., Pang, S. L., \& Chiu, H. F. (2010). Lived experience of caregivers of persons with dementia in Hong Kong: A qualitative study. East Asian Arch Psychiatry, 20(4), 163-168.

Cruz, D. C. M., Loureiro, H. A. M., Silva, M. A. N. C. G. M. M., \& Fernandes, M. M. (2010). Revisão SistemáAs vivências do cuidador informal do idoso dependente. Revista de Enferma- tica da Literatura gem Referência, 3(2), 127-136.

Innes, A., Morgan, D., \& Kostineuk, J. (2011). Dementia care in rural and remote set- Revisão Sistemátings: A systematic review of informal/family caregiving. Maturitas, 68(1), 34-46. tica da Literatura

Stenberg, U., Ruland, C. M., \& Miaskowski, C. (2010). Review of the literature on the Revisão Sistemáeffects of caring for a patient with cancer. Psycho-Oncology, 19(10), 1013-1025. tica da Literatura

Washington, K. T., Meadows, S. E., Elliott, S. G., \& Koopman, R. J. (2011). Information Revisão Sistemáneeds of informal caregivers of older adults with chronic health conditions. tica da Literatura Patient Education and Couseling, 83(1), 37-44.

Blake, H. (2008). Caregiver stress in traumatic brain injury. International Journal of Revisão da LiteTherapy and Rehabilitation, 15(6), 263-271.

$$
\text { ratura }
$$

Jorgensen, D., Arksey, H., Parsons, M., \& Jacobs, S. (2009). Caregiver assessment of support need, reaction to care, and assessment of depression. Home Health Care

Estudo Misto

IV

IV

IV

IV

V

V

V

IV

V

I

I

I

I

V

IV Services Quarterly, 28(4), 130-150.

Esta revisão é composta por 10 estudos quantitativos, 5 qualitativos, 4 revisões sistemáticas da literatura, 1 revisão da literatura e 1 estudo misto. Tendo em consideração o nível de evidência, podemos salientar que 5 dos artigos se enquadram no nível V, 9 no nível IV, 3 no nível III e IV são do nível de evidência mais elevado, nível I.

Tendo em consideração a transição inerenteà integração do papel de cuidador familiar, a identificação das necessidades mais evidenciadas por estes, no desem- penho desta atividade e o seu correto diagnóstico pode ser, de certo modo, um ponto de viragem em todo o processo de transição. Desta forma, as necessidades podem ser consideradas como fatores inibidores e/ ou facilitadores, em parte devido ao modo como são vivenciadas/experienciadas, diagnosticadas e satisfeitas/ suprimidas. Com um conjunto de intervenções de enfermagem devidamente planeadas, concertadas e eficazes, pretende-se que estas necessidades sejam satisfeitas de modo a que, no final, se obtenha um 
conjunto de indicadores de resultados positivos, designadamente a mestria e a integração fluida do novo papel. Se a necessidade for um fator inibidor ao qual o enfermeiro esteja atento e intervenha, este pode deixar de existir com as corretas intervenções e o cuidador continua na sua transição situacional saudável com a aquisição das competências necessárias.

Este trabalho permite evidenciar as necessidades manifestadas com maior frequência na literatura científica, no sentido de facultar uma orientação possível para a prática de enfermagem com maior qualidade e eficiência, com planos de cuidados individualizados, contextualizados e direcionados para os cuidadores e, neste sentido, promover o seu bem estar e melhorar os cuidados que prestam à pessoa dependente. Os cuidadores familiares são parceiros extremamente importantes para garantir a continuidade de cuidados imprescindíveis para a pessoa dependente, pelo que os enfermeiros devem ter uma especial atenção para garantir a manutenção eficaz desta parceria.

Tal como é realçado na análise desta revisão da literatura, as necessidades dos cuidadores familiares demonstradas mais frequentemente são diversificadas. Assim, e tendo em consideração o assumir de um novo papel e as mudanças que são operadas, consideramos pertinente agrupar as mesmas à luz da Teoria das Transições, como fatores facilitadores ou dificultadores da transição (recursos comunitários e sociais, conhecimentos e aprendizagem de habilidades, significado pessoal, crenças e atitudes e, por último, condição socieconómica) do novo papel de prestação de cuidados e a sua continuidade, os quais abordaremos de seguida.

\section{Recursos Comunitários e Sociais}

A necessidade de apoio familiar e social é uma das mais salientadas pelos diferentes autores, pelo que o apoio para colmatar esta necessidade pode ser considerado uma condição facilitadora em todo o processo.

A família é determinante em toda a transição para o papel de cuidador familiar, pois desempenha uma importante função em todo o processo. Um bom relacionamento familiar, tanto entre familiares, como entre cuidador e pessoa dependente, é um dos aspetos mais evidentes nos trabalhos incluídos nesta revisão, pois promove o estabelecimento de uma rede de apoio adequado e necessário no seio familiar (Francis, Bowman, Kypriotakis, \& Rose, 2011; Mavundla, Toth, \& Mphelane, 2009; Molassiotis, Wilson, Blair, Howe, \& Cavet, 2009; Stenberg, Ruland, \& Miaskowski, 2010; Blake, 2008; Jorgensen, Arksey, Parsons, \& Jacobs, 2009).

Tal como refere Francis et al. (2011), é essencial intervir e disponibilizar estratégias eficazes que permitam o desenvolvimento, a manutenção e o fortalecimento destas relações, pela sua importância em todo o processo.

Stenberg, Ruland, e Miaskowski (2010), numa revisão sistemática sobre os efeitos da prestação de cuidados a pessoas com cancro, referem que um dos aspetos positivos do cuidar é o tempo que as famílias passam juntas e a qualidade desses momentos.

O apoio social é também referido por inúmeras ocasiões, no sentido de evitar o isolamento social (Robison, Fortinsky, Kleppinger, Shugrue, \& Porter, 2009; Mavundla et al., 2009; Molassiotis et al., 2009; Chan, Mok, Wong, Pang, \& Chiu, 2010; Cruz, Loureiro, Silva, \& Fernandes, 2010; Blake, 2008; Jorgensen et al., 2009), facilitando o acesso aos recursos disponíveis na comunidade e sociedade e o auxílio inerente aos grupos de apoio.

A ausência de tempo que o cuidador dispõe para si próprio é tido como um fator inibidor, devido aos recursos insuficientes que permitam pausas na prestação de cuidados, para assim dispor de tempo para as suas necessidades individuais, tanto de lazer como de socialização. Diversos autores destacam a relevância dos grupos de apoio (Sinclair, Armes, Randhawa, \& Bayer, 2010; Ganea et al., 2010; Mavundla et al., 2009; Chan et al., 2010; Jorgensen et al., 2009), ou a necessidade de intervenções e/ou serviços que permitem a pausa e o descanso na atividade de cuidar (Casado, 2008; Carretero, Garcés, \& Ródenas, 2006; Salin, Kaunone, \& Astedt-Kurki, 2009; Woolfe, McMillan, \& Conway, 2007; Cruz et al., 2010), sendo todos estes considerados como fatores facilitadores e fundamentais para a manutenção de um bom nível de saúde do cuidador.

As necessidades evidenciadas a nível dos recursos da comunidade e sociedade, quando devidamente satisfeitas, permitem reforçar o relacionamento interpessoal, tanto no seio familiar como comunitário, promovendo ainda o desenvolvimento de estratégias individuais facilitadoras do aumento da auto estima, fortalecimento da identidade pessoal, permitindo assim integrar melhor o papel de cuidador. 


\section{Conhecimentos e aprendizagem e preparaçáo}

Dotar os cuidadores da pessoa dependente dos conhecimentos e capacidades que precisam é importante, não só para cuidarem melhor dos seus familiares, como de si mesmos, sendo um fator facilitador para um desempenho das funções de um modo mais saudável.

O apoio dos enfermeiros, enquanto profissionais de saúde habilitados para transmitir a informação necessária ao desempenho do novo papel, assim como a aprendizagem e aquisição de competências, são realçadas por diferentes investigadores (Salin et al., 2009; Sinclair et al., 2010; Davey, Wiles, Asburn, \& Murphy, 2004; Molassiotis et al., 2009; Chan et al., 2010; Jorgensen et al., 2009).

Além deste aspeto, também é destacado o acesso aos serviços de saúde (Chan et al., 2010; Innes, Morgan, \& Kostineuk, 2011) que, devido a todo um conjunto de dificuldades e adversidades (aspetos burocráticos, barreiras arquitetónicas, recursos humanos reduzidos, inexistência de protocolos e articulação entre serviços, entre outros), podem tornar-se elementos inibidores do processo de assunção do papel de cuidadores, caso não sejam devidamente superadas.

Relativamente aos conhecimentos a adquirir pelo cuidador, estes devem ser específicos e permitir a aquisição de determinadas competências instrumentais úteis na prestação dos cuidados. Podem também ser transmitidos com o intuito de melhorar competências já adquiridas, através da aprendizagem de alguns procedimentos e técnicas elementares mas importantes para alguns cuidados específicos a serem prestados á pessoa dependente (Ryn et al., 2011; Ganea et al., 2010; Davey et al., 2004; Cruz et al., 2010).

Alguns autores destacam a importância destas competências instrumentais na prestação de cuidados, designadamente Ryn et al. (2011), que salientam a importância do treino para cuidar, do mesmo modo Ganea et al. (2010) e Cruz et al. (2010), que realçam um conjunto de habilidades que os cuidadores precisam adquirir no domínio dos autocuidados para satisfazerem todas atividades de vida diária.

O domínio dos conhecimentos é para os cuidadores familiares uma das necessidades mais importantes, pois a satisfação desta permite-lhes a melhor perceção de toda a situação vivenciada e, consequentemente, do seu novo papel e da sua relevância. Neste contexto, é importante que os enfermeiros compreendam a complexidade da transmissão de informação ao cuidador e da consequente integração como conhecimento, para assim permitir a sua perceção eficaz e, deste modo, evitar que seja considerado um fator inibidor (Nichols, Martindale-Adamsb, Greeneb, \& Burnsc, 2009; Ganea et al., 2010; Jullamate, Azeredo, Paúl, \& Subgranon, 2006; Molassiotis et al., 2009; Chan et al., 2010). Assim, o enfermeiro deverá conhecer devidamente os recursos do cuidador, tanto pessoais como sociais, para poder ajustar as suas intervenções e otimizá-las. A informação deve ser transmitida de forma clara, em ambiente calmo, sobretudo quando a mesma incide sobre a doença que afeta o seu familiar, a sua evolução, o seu prognóstico e os recursos disponíveis, tal como é destacado por Chan et al. (2010) e Ganea et al. (2010).

Stenberg et al. (2010) demonstram que a necessidade de informação difere mediante o estádio da doença, mas salientam a sua a importância, não só pelos dados disponibilizados sobre a mesma, mas também sobre o prognóstico/evolução, pelas opções de tratamento, o que confere uma certa sensação de controlo e segurança ao cuidador familiar. Washington, Meadows, e Elliot (2011) elaboraram uma revisão sistemática no domínio específico da informação, realçando a necessidade de informação geral, sobre etiologia, diagnóstico e prognóstico da doença, assim como dos apoios e ajudas que dispõe, mas também específica nomeadamente educação e informação percetível e individualizada, e separadamente da pessoa doente. Nichols et al. (2009), num trabalho sobre as preocupações mais evidenciadas pelos cuidadores da pessoa com demência, realçam que as necessidades de informação dos cuidadores varia mediante a presença de depressão e também da gravidade da demência apresentada pela pessoa de quem cuidam.

\section{Significado Pessoal, Crenças e Atitudes}

O papel de cuidador familiar é extremamente desgastante, provocando um aumento de sobrecarga emocional. Nesta perspetiva, torna-se evidente a relevância do apoio emocional e o papel que este ocupa em todo o processo de transição e na integração saudável deste papel (Robinson et al., 2009; Woolfe et al., 2007; Ganea et al., 2010; Molassiotis et al., 2009; Cruz et al., 2010; Stenberg et al., 2010). No mesmo sentido, diversos autores destacam a importância da aquisição 
de estratégias de coping eficazes (Ryn et al., 2011; Molassiotis et al., 2009; Chan et al., 2010; Blake, 2008; Jorgensen et al., 2009), podendo ser uma importante condição facilitadora na prestação de cuidados e no transpor das dificuldades que daí advêm, quando a esta necessidade equivale intervenções eficazes.

Segundo Molassiotis et al. (2009), a família é um importante recurso para o cuidador, não só pelas relações interpessoais, mas também pelo apoio psicoemocional que disponibiliza. Este aspeto é também realçado por Chan et al. (2010), que demonstra que a falta de apoio emocional e assistência por parte dos familiares, assim como o não reconhecimento do seu papel enquanto cuidador, pode dificultar todo o processo de transição.

Vários autores, tais como Cruz et al. (2010), Stenberg et al. (2010) e Blake (2008) destacam também a necessidade de apoio emocional, importante para gerir melhor os diferentes tipos de reações emocionais, tais como ansiedade, medo e incerteza. Este aspeto deve-se ao fato de que as estratégias de coping a que os cuidadores mais recorrem serem usualmente centradas nas emoções, realçando, contudo, a importância de estratégias que permitam a resolução de problemas, tais como a aprendizagem de formas de lidar com o stress, sentimentos, medos, solidão, de modo a diminuir o stress do cuidador (Salin et al., 2009; Woolfe et al., 2007; Blake, 2008). Outros autores, nomeadamente Ryn et al. (2011) Jorgensen et al. (2009), Molassiotis et al. (2009) e Cruz et al. (2010) destacam a importância da aquisição e promoção de estratégias de coping que sejam eficazes para a resolução de problemas, centradas nos mesmos e não na vertente emocional.

Woolfe et al. (2007) realça as necessidades psicológicas dos cuidadores familiares, bem como do apoio emocional como agentes facilitadores, evidenciando a aprendizagem de formas para lidar com o stress de forma eficaz e saudável.

Ainda neste domínio é reconhecido o papel de cuidador familiar, dando-se destaque às razões que levaram ao assumir deste papel, assim como à importância para a pessoa dependente (Chan et al., 2010; Jorgensen et al., 2009).

Num estudo realizado no âmbito do cuidado informal a pessoas com doença mental Mavundla et al. (2009) demonstrou que, para além da importância do envolvimento familiar já salientado, também o apoio social o é, de forma a evitar o isolamento a que são sujeitos os cuidadores familiares e as suas famílias em inúmeras situações, maioritariamente pelo estigma social inerente a este tipo de patologia, devido às crenças previamente definidas a nível social e pelo défice de conhecimento neste domínio.

\section{Condição Socioeconómica}

Relativamente a esta condição diversos autores referem a sobrecarga financeira e os custos do cuidado informal que emerge dos cuidados à pessoa dependente, pelos elevados custos que lhe estão associados, sendo considerados fatores dificultadores, destacando assim a necessidade de apoio económico que daí advêm (Casado, 2008; Woolfe et al., 2007; Ryn et al., 2011; Sinclair et al., 2010; Mavundla et al., 2009; Cruz et al., 2010; Stenberg et al., 2010; Jorgensen et al., 2009).

Ainda neste âmbito, alguns autores mencionam a necessidade da flexibilidade em termos de horário laboral, para assim conseguirem conciliar o cuidado prestado à pessoa dependente com o emprego e a fonte de rendimento que precisam de manter (Robinson et al., 2009; Ryn et al., 2011; Jorgensen et al., 2009).

Esta revisão da literatura apresenta algumas limitações. Assim, ao recorrer ao termo de pesquisa cuidador familiar, alguns trabalhos poderão ter sido excluídos, mesmo abordando esta temática ou quando o cuidado informal é prestado por um elemento que não familiar, visto que o termo usualmente utilizado na literatura é cuidador informal (informal caregiver).

Por outro lado, organizar as necessidades evidenciadas pelos cuidadores familiares nos diferentes trabalhos científicos com o recurso à Teoria das Transições pode dificultar a sua categorização, pelo que algumas podem ser incluídas em diferentes categorias ou individualizadas em categorias específicas.

Tendo em consideração as produções científicas produzidas e o interesse desta temática no contexto português, torna-se interessante elaborar o mesmo tipo de análise, partindo dos mesmos termos de pesquisa, mas na realidade portuguesa.

\section{Conclusão}

O reconhecimento das necessidades dos cuidadores familiares é de extrema importância, tendo em consideração que é a primeira etapa para o 
diagnóstico situacional e posterior planeamento das intervenções de enfermagem. Uma recolha de dados eficaz permite um correto diagnóstico destas necessidades e, se for associado a um plano de intervenções que se esperam úteis, poderá auxiliar todo o processo de transição. A ausência de intervenção pode culminar numa transição não saudável e, assim, resultar em prejuízos para a saúde, tanto do cuidador familiar como, em consequência, do próprio familiar dependente. Na análise das diversas produções científicas, identificaram-se diferentes tipos de necessidades, em diversos contextos. Tendo em consideração estas necessidades criaram-se as seguintes categorias: recursos comunitários e sociais, conhecimentos e aprendizagem de habilidades, significado pessoal, crenças e atitudes e, por último, condição socieconómica. Apesar dos estudos científicos integrados nesta revisão da literatura terem sido levados a cabo em diferentes domínios e tendo em consideração a singularidade dos mesmos, as necessidades evidenciadas são, de certo modo, transversais a todos. Assim sendo, pode referir-se que, sem minimizar a individualidade deste processo, a transição inerente à integração do papel de cuidador familiar é, de um modo geral, universal, pois dele resultam necessidades em tudo semelhantes a todas as transições deste género.

Um dos aspetos mais destacados nesta revisão referese à importância da família, assim como o apoio social, em todo este processo de transição para os cuidadores familiares. Com um bom relacionamento no seio familiar, a rede de apoio informal é realmente melhorada e fortalecida, o que permite satisfazer uma das necessidades mais demonstradas. Uma outra bem evidente reporta-se ao apoio psicoemocional, assim como a aquisição de estratégias de coping eficazes que, sendo potenciadoras da resolução eficaz de problemas, permitem ao cuidador familiar uma melhor integração do seu novo papel. Vários estudos salientam igualmente o apoio de profissionais de saúde, designadamente de enfermeiros, para a aprendizagem de competências instrumentais que facultem ao cuidador familiar prestar melhores cuidados. Finalmente, a necessidade de informação por parte dos cuidadores familiares é transversal a todos os estudos integrados nesta revisão da literatura, em diferentes domínios, tanto ao nível da doença como do cuidado a prestar.

Deixamos como recomendação que, tendo em consideração que parte significativa dos estudos que integram esta revisão foram realizados em contextos diversificados, deveria ser realizado trabalho semelhante no contexto português, eventualmente em domínios específicos, como necessidades dos cuidadores de pessoas vítimas de Acidente Vascular Cerebral (AVC) ou com limitações físicas, de pessoas com Alzheimer ou Parkinson, no sentido de uma melhor perceção das necessidades singulares evidenciadas por estes cuidadores familiares.

\section{Referências bibliograficas}

Carrilho, M. J., \& Patrício, L. (2008). A situação demográfica recente em Portugal. Revista dos Estudos Demográficos, 44, 35-80.

Chick, N., \& Meleis, A. I. (2010). Transitions: A nursing concern. In A. I. Meleis (Ed.), Transitions theory: Middle range and situation specific theories in nursing research and practice (pp. 24-38). New York, NY: Springer.

Cruz, D. C. M., Loureiro, H. A. M., Silva, M. A. N. C. G. M. M., \& Fernandes, M. M. (2010). As vivências do cuidador informal do idoso dependente. Revista de Enfermagem Referência, 3(2), 127-136. doi:10.12707/RIII1018

Instituto Nacional de Estatística. (2012). Censos 2011: Resultados definitivos - Portugal: XV recenseamento geral da população: $V$ recenseamento geral da habitação. Lisboa, Portugal: Autor.

International Council of Nurses. (2011). CIPE versão 2: Classificação internacional para prática de enfermagem (H. Castro, Trad.). Lisboa, Portugal: Ordem dos Enfermeiros.

Lewin, R. F., Singleton, J. K., \& Jacobs, S. K. (2008). Developing and evaluating clinical practice guidelines: A systematic approach. In E. Capezuti, D. Zwicker, M. Mezey, \& T. Fulmer (Eds.), Evidence-based geriatric nursing protocols for best practice (3rd ed., pp. 1-8). New York, NY: Springer.

Martins, R. M. L. (2008). A relevância do apoio social na velhice. Educação, Ciência e Tecnologia, 128-134.

Meleis, A. I. (2010). Transitions theory: Middle range and situation specific theories in nursing research and practice. New York, NY: Springer.

Sarmento, E., Pinto, P., \& Monteiro, S. (2010). Cuidar do idoso: Dificuldades dos familiares. Coimbra, Portugal: Formasau.

Schumacher, K., \& Meleis, A. I. (2010). Transitions: A central concept in nursing. In A. I. Meleis (Ed.), Transitions theory: Middle range and situation specific theories in nursing research and practice (pp. 38-51). New York, NY: Springer.

Sequeira, C. (2010). Cuidar de idosos com dependência física e mental. Lisboa, Portugal: Lidel. 
